\title{
Phase III Randomized Trial of Chemotherapy With or Without Bevacizumab in Patients With Recurrent or Metastatic Head and Neck Cancer.
}

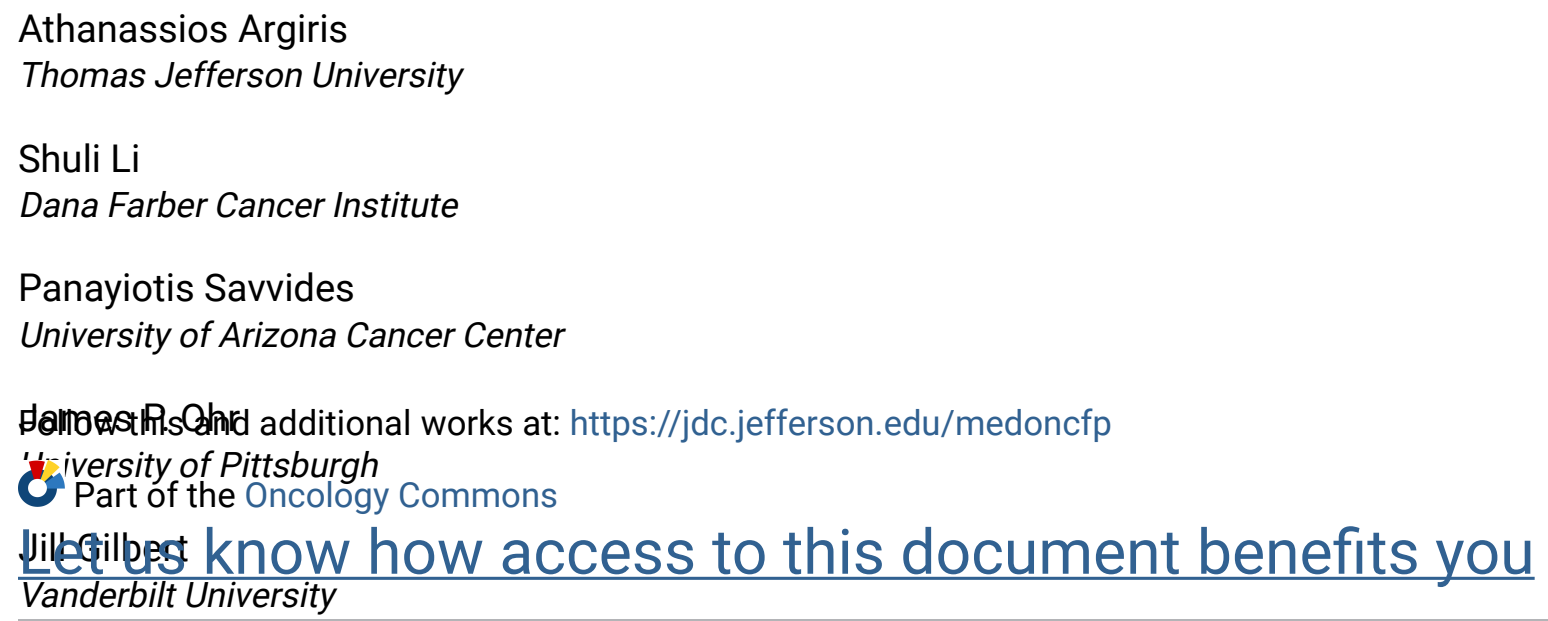

\section{Recommended Citation}

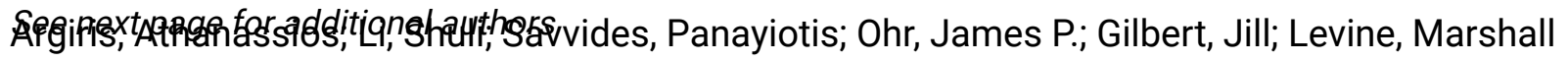
A.; Chakravarti, Arnab; Haigentz, Missak; Saba, Nabil F.; Ikpeazu, Chukwuemeka V.; Schneider, Charles J.; Pinto, Harlan A.; Forastiere, Arlene A.; and Burtness, Barbara, "Phase III Randomized Trial of Chemotherapy With or Without Bevacizumab in Patients With Recurrent or Metastatic Head and Neck Cancer." (2019). Department of Medical Oncology Faculty Papers. Paper 106. https://jdc.jefferson.edu/medoncfp/106

This Article is brought to you for free and open access by the Jefferson Digital Commons. The Jefferson Digital Commons is a service of Thomas Jefferson University's Center for Teaching and Learning (CTL). The Commons is a showcase for Jefferson books and journals, peer-reviewed scholarly publications, unique historical collections from the University archives, and teaching tools. The Jefferson Digital Commons allows researchers and interested readers anywhere in the world to learn about and keep up to date with Jefferson scholarship. This article has been accepted for inclusion in Department of Medical Oncology Faculty Papers by an authorized administrator of the Jefferson Digital Commons. For more information, please contact: JeffersonDigitalCommons@jefferson.edu. 


\section{Authors}

Athanassios Argiris, Shuli Li, Panayiotis Savvides, James P. Ohr, Jill Gilbert, Marshall A. Levine, Arnab Chakravarti, Missak Haigentz, Nabil F. Saba, Chukwuemeka V. Ikpeazu, Charles J. Schneider, Harlan A. Pinto, Arlene A. Forastiere, and Barbara Burtness 


\title{
Phase III Randomized Trial of Chemotherapy With or Without Bevacizumab in Patients With Recurrent or Metastatic Head and Neck Cancer
}

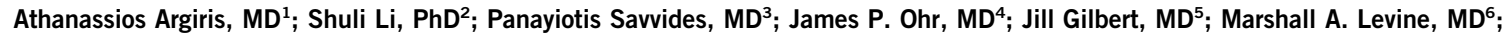 \\ Arnab Chakravarti, MD; Missak Haigentz Jr, MD ${ }^{8}$; Nabil F. Saba, MD ${ }^{9}$; Chukwuemeka V. Ikpeazu, MD, PhD ${ }^{10}$; Charles J. Schneider, MD ${ }^{11}$; \\ Harlan A. Pinto, MD ${ }^{12}$; Arlene A. Forastiere, MD ${ }^{13}$; and Barbara Burtness, MD ${ }^{14,15}$
}

PURPOSE We evaluated the addition of bevacizumab, a humanized monoclonal antibody that targets vascular endothelial growth factor, to platinum-based chemotherapy in recurrent or metastatic squamous cell carcinoma of the head and neck (SCCHN).

PATIENTS AND METHODS Patients with chemotherapy-naive (or with prior platinum as part of multimodal therapy completed $\geq 4$ months earlier) recurrent or metastatic SCCHN were randomly assigned to receive a platinumbased chemotherapy doublet with or without bevacizumab $15 \mathrm{mg} / \mathrm{kg}$ given intravenously every 3 weeks until disease progression. Chemotherapy could be discontinued after six cycles if a maximum response was achieved.

RESULTS The study randomly assigned 403 patients. Median overall survival (OS) was 12.6 months with bevacizumab plus chemotherapy (BC) and 11.0 months with chemotherapy alone (hazard ratio, $0.87 ; 95 \% \mathrm{Cl}$, 0.70 to $1.09 ; P=.22$ ). At 2,3 , and 4 years, the OS rates were $25.2 \% v 18.1 \%, 16.4 \% v 10.0 \%$, and $11.8 \% v$ $6.4 \%$ for $\mathrm{BC}$ versus chemotherapy, respectively. In an analysis of 365 eligible patients who started treatment, the hazard ratio was $0.82(95 \% \mathrm{Cl}, 0.65$ to $1.04 ; P=.10)$, with a median OS of 14.2 months on $\mathrm{BC} v 11.1$ months on chemotherapy. Median progression-free survival with BC was 6.0 months $v 4.3$ months with chemotherapy ( $P=.0014)$. Overall response rates were $35.5 \%$ with $\mathrm{BC}$ and $24.5 \%$ with chemotherapy $(P=.016)$. There was increased toxicity, including a higher rate of treatment-related grade 3 to 5 bleeding events $(6.7 \% v 0.5 \%$; $P<$ $.001)$ and treatment-related deaths $(9.3 \% \vee 3.5 \% ; P=.022)$ with $\mathrm{BC}$ versus chemotherapy.

CONCLUSION The addition of bevacizumab to chemotherapy did not improve OS but improved the response rate and progression-free survival with increased toxicities. These results encourage biomarker-driven studies of angiogenesis inhibitors with better toxicity profiles in select patients with SCCHN.

J Clin Oncol 37:3266-3274. @ 2019 by American Society of Clinical Oncology

\section{INTRODUCTION}

Historically, $>50 \%$ of patients with squamous cell carcinoma of the head and neck (SCCHN) develop disease recurrence in either local or distant sites after potentially curative treatment with surgery and/or radiation with or without chemotherapy. The cornerstone of treatment in recurrent or metastatic SCCHN remains platinum-based doublet chemotherapy with a reported median overall survival (OS) of 6 to 8 months. ${ }^{1}$ Regimens with a platinum plus a taxane (paclitaxel or docetaxel) often are used as treatment options in this setting. ${ }^{2}$ Until the introduction of immune checkpoint inhibitors, cetuximab was the only molecularly targeted therapy with proven benefit in first-line treatment of recurrent or metastatic SCCHN. The addition of cetuximab to platinum (cisplatin or carboplatin) and fluorouracil (FU) improved OS, progression-free survival (PFS), and the objective response rate compared with platinum or $\mathrm{FU}$ alone. ${ }^{3}$ However, the 2-year OS rate remained poor at $14 \%$.
Angiogenesis plays a critical role in the development and growth of SCCHN and has emerged as an important target for anticancer therapy. ${ }^{4}$ Increased expression of vascular endothelial growth factor (VEGF), a potent inducer of angiogenesis, has been linked to poor prognosis in SCCHN. ${ }^{5}$ A meta-analysis concluded that patients whose primary tumors overexpressed VEGF, as measured by immunochemistry, had a 1.88fold higher mortality. ${ }^{6}$

Bevacizumab is an anti-VEGF, humanized monoclonal antibody that has been used in the treatment of several advanced solid tumors, including colorectal, non-smallcell lung, ovarian, and cervical cancers, in combination with chemotherapy. Preclinical studies in SCCHN support the combination of a taxane and bevacizumab. ${ }^{7}$ Paradoxically, bevacizumab may stabilize and mature tumor vasculature, which leads to lower interstitial fluid pressure and increased tumor blood flow. This may reduce tumor hypoxia and lead to improved delivery of chemotherapy to tumor tissue, thereby providing

\section{ASCO}


a potential mechanism for the synergistic effect of bevacizumab with other systemic agents. ${ }^{4,8}$ In the clinic, a number of phase II trials have explored bevacizumab-based combinations in recurrent or metastatic SCCHN. ${ }^{9-11}$ A study that investigated the combination of pemetrexed and bevacizumab reported promising efficacy results with a median OS of 11.3 months but with a relatively high rate of hemorrhagic complications ( $15 \%$ with grade $\geq 3$ ). ${ }^{9}$ On the other hand, a clinical trial of cetuximab and bevacizumab in recurrent or metastatic SCCHN did not report a concerning rate of hemorrhage. ${ }^{10}$

We conducted a phase III study (E1305; ClinicalTrials.gov identifier: NCT00588770) coordinated by the Eastern Cooperative Oncology Group (ECOG)-American College of Radiology Imaging Network Cancer Research Group that compared investigator's-choice platinum-based doublet with or without bevacizumab in patients with recurrent or metastatic SCCHN. The primary objective of this study was to evaluate the OS.

\section{PATIENTS AND METHODS}

\section{Eligibility}

Adult patients with an ECOG performance status of 0 to 1 and adequate end organ function with pathologically confirmed SCCHN from any primary site, including unknown primary lymphadenopathy of the head and neck, were eligible; those with nonkeratinizing nasopharyngeal carcinoma or squamous cell carcinoma that originated in the skin were excluded. SCCHN was either metastatic or recurrent and judged incurable by surgery or radiation. Patients who refused radical surgery for recurrent disease were eligible. Measurable disease by RECIST with baseline scans obtained $\leq 4$ weeks before randomization was required. No prior chemotherapy or biologic/moleculartargeted therapy for recurrent or metastatic SCCHN was permitted. Prior chemotherapy or cetuximab as part of initial potentially curative therapy was allowed if completed $\geq 4$ months previously and if patients had remained progression free for at least 4 months after completion of this therapy. Patients with tumors that unequivocally invaded major vessels or central cavitary lung metastases were excluded. Patients were not permitted to have a history of thrombosis requiring anticoagulation, bleeding associated with SCCHN, or gross hemoptysis. No prior treatment with bevacizumab was allowed. A maximum of one prior radiotherapy regimen, curative or palliative, to the head and neck was allowed. Furthermore, the trial excluded patients who received chronic daily treatment with aspirin or nonsteroidal anti-inflammatory agents known

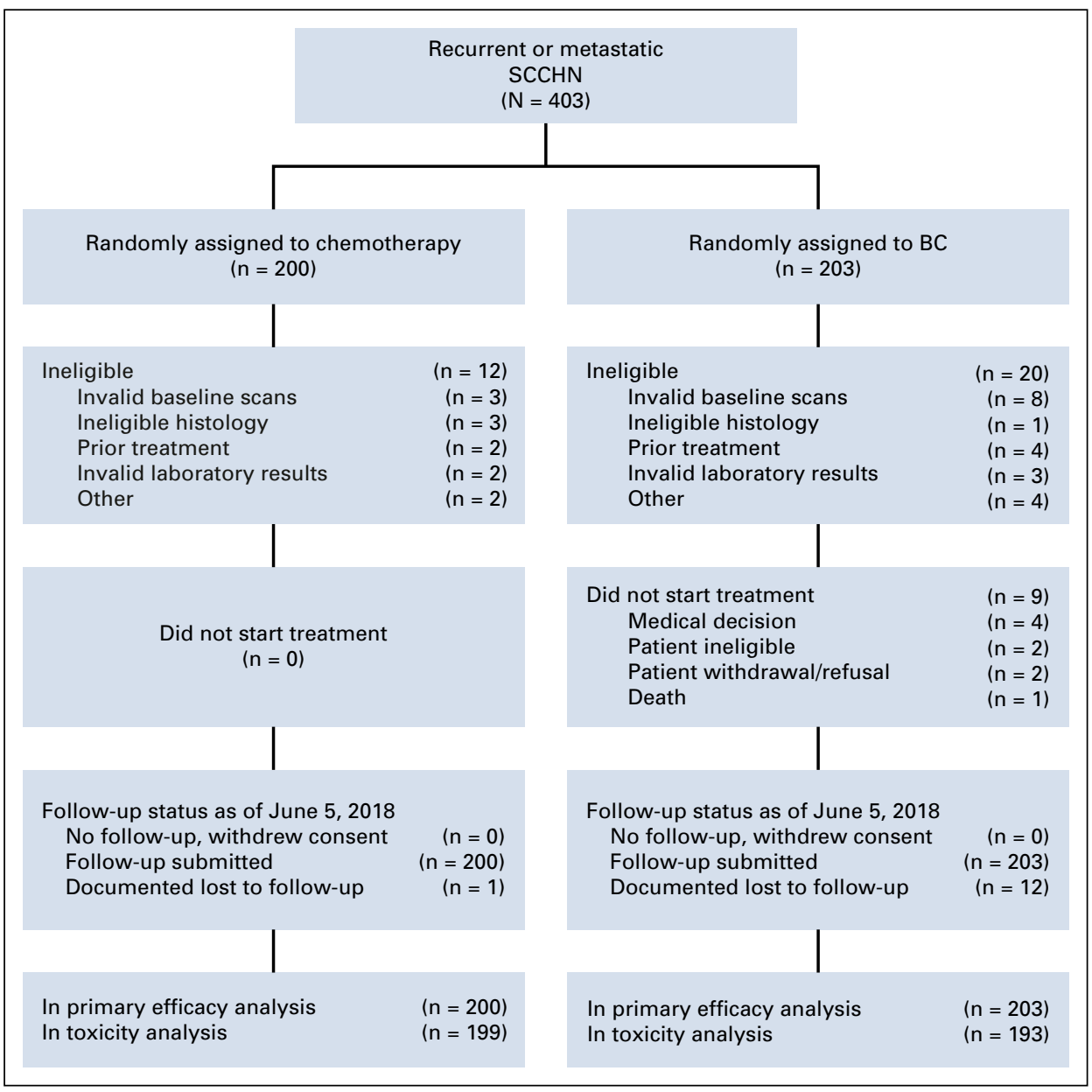

FIG 1. CONSORT diagram of study E1305. BC, bevacizumab plus chemotherapy; SCCHN, squamous cell carcinoma of the head and neck. 
TABLE 1. Patient Characteristics

\begin{tabular}{|c|c|c|c|c|c|c|}
\hline \multirow[b]{2}{*}{ Characteristic } & \multicolumn{2}{|c|}{$\begin{array}{l}\text { Chemotherapy } \\
(n=200)\end{array}$} & \multicolumn{2}{|c|}{$B C(n=203)$} & \multicolumn{2}{|c|}{ Total $(\mathrm{N}=403)$} \\
\hline & No. & $\%$ & No. & $\%$ & No. & $\%$ \\
\hline Male & 168 & 84.0 & 176 & 86.7 & 344 & 85.4 \\
\hline Female & 32 & 16.0 & 27 & 13.3 & 59 & 14.6 \\
\hline$\geq 70$ & 21 & 10.5 & 27 & 13.3 & 48 & 11.9 \\
\hline \multicolumn{7}{|l|}{ Race } \\
\hline White & 168 & 87.0 & 179 & 89.9 & 347 & 88.5 \\
\hline Black & 21 & 10.9 & 18 & 9.0 & 39 & 9.9 \\
\hline Not reported & 7 & & 4 & & 11 & \\
\hline \multicolumn{7}{|l|}{ Chemotherapy assignment } \\
\hline Cisplatin plus docetaxel & 95 & 47.5 & 99 & 48.8 & 194 & 48.1 \\
\hline Carboplatin plus docetaxel & 80 & 40.0 & 78 & 38.4 & 158 & 39.2 \\
\hline Cisplatin plus fluorouracil & 14 & 7.0 & 17 & 8.4 & 31 & 7.7 \\
\hline Carboplatin plus fluorouracil & 11 & 5.5 & 9 & 4.4 & 20 & 5.0 \\
\hline \multicolumn{7}{|l|}{ Ethnicity } \\
\hline Hispanic & 11 & 5.9 & 11 & 5.6 & 22 & 5.7 \\
\hline Hypopharynx & 11 & 5.5 & 4 & 2.0 & 15 & 3.7 \\
\hline Larynx & 45 & 22.5 & 46 & 22.7 & 91 & 22.6 \\
\hline Salivary glands & 4 & 2.0 & 1 & 0.5 & 5 & 1.2 \\
\hline Paranasal sinuses & 3 & 1.5 & 2 & 1.0 & 5 & 1.2 \\
\hline Nose & 1 & 0.5 & 1 & 0.5 & 2 & 0.5 \\
\hline No primary identified & 5 & 2.5 & 3 & 1.5 & 8 & 2.0 \\
\hline Other & 3 & 1.5 & 4 & 2.0 & 7 & 1.7 \\
\hline Multiple primary & 6 & 3.0 & 9 & 4.4 & 15 & 3.7 \\
\hline \multicolumn{7}{|l|}{ Histologic grade } \\
\hline Well differentiated & 14 & 7.0 & 18 & 8.9 & 32 & 7.9 \\
\hline Moderately differentiated & 87 & 43.5 & 82 & 40.4 & 169 & 41.9 \\
\hline Poorly differentiated & 73 & 36.5 & 73 & 36.0 & 146 & 36.2 \\
\hline Undifferentiated & 2 & 1.0 & 8 & 3.9 & 10 & 2.5 \\
\hline Grade cannot be assessed & 24 & 12.0 & 22 & 10.8 & 46 & 11.4 \\
\hline
\end{tabular}


TABLE 1. Patient Characteristics (continued)

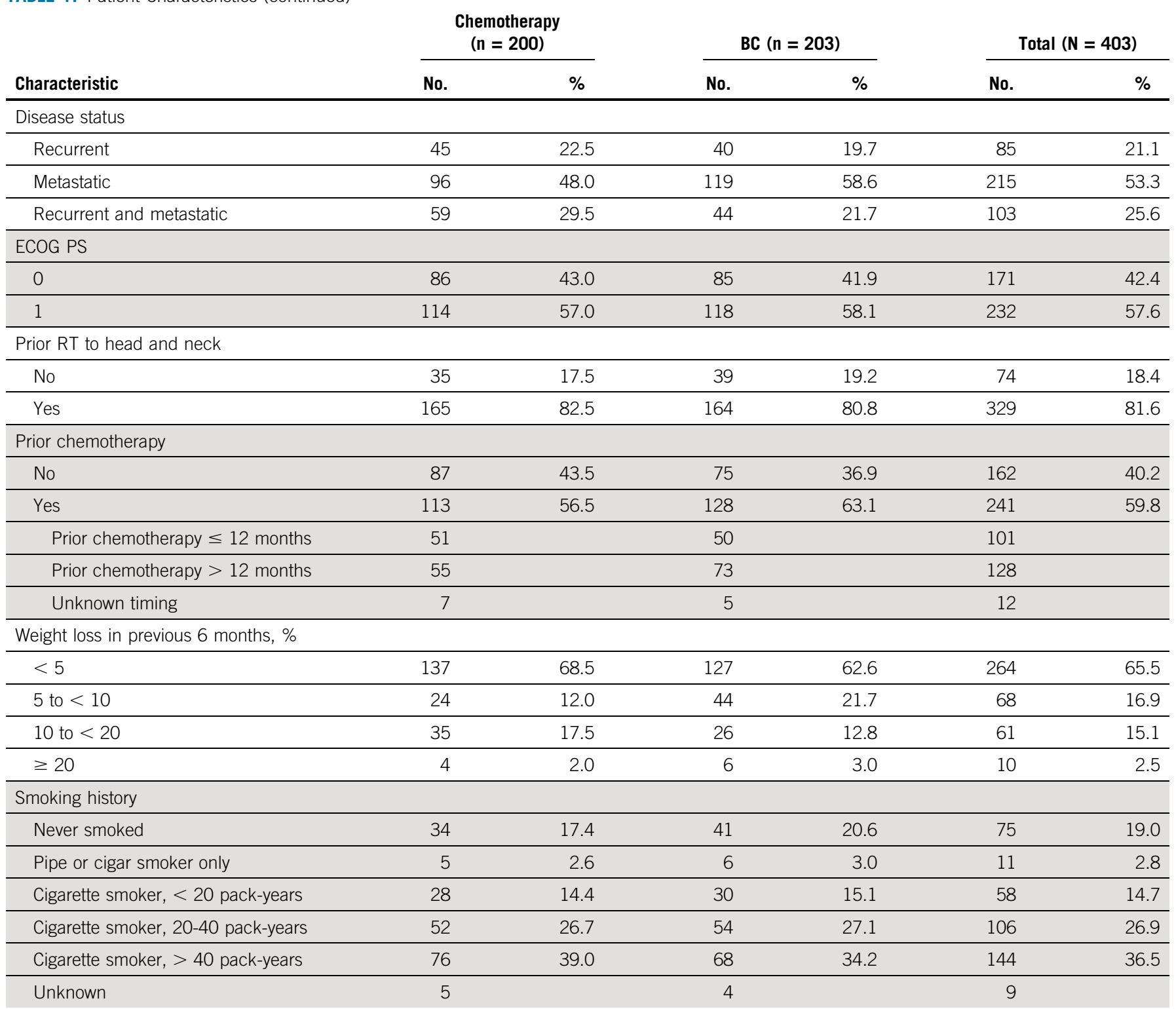

Abbreviations: BC, bevacizumab plus chemotherapy; ECOG PS, Eastern Cooperative Oncology Group performance status; RT, radiotherapy.

to inhibit platelet function, patients with uncontrollable hypertension or active heart disease, and patients with hypercalcemia related to head and neck cancer.

All patients provided written informed consent. The study was approved by the ethics committee of each participating center and was conducted in compliance with the Declaration of Helsinki.

\section{Study Design}

Patients were equally assigned randomly between the two treatment arms. On the basis of a previous analysis of ECOG-American College of Radiology Imaging Network trials, ${ }^{12}$ stratification factors were as follows: chemotherapy regimen, performance status, prior radiation to the head and neck, and weight loss in the past 6 months $(<5 \% \mathrm{~V}$ $\geq 5 \%$ ). Randomization was done using permuted blocks within strata, with dynamic balancing within main institutions and their affiliate networks. The primary study end point was OS. Secondary efficacy end points were PFS, objective response, and toxicity.

The design called for a total accrual of 400 patients to give full information of 354 deaths to provide $80 \%$ power to detect a $26 \%$ reduction in the hazard rate (corresponding to an improvement in median survival from 8.5 to 11.5 months) using a one-sided test with an overall type I error rate of $2.5 \%$. Interim analyses were planned for all semiannual data safety monitoring committee meetings, which started when $25 \%$ of the information (89 deaths) was available. The trial was monitored according to principles of group sequential methods using a one-sided 
O'Brien-Fleming upper boundary to preserve the overall type I error of 0.025 . The rates of grade 3 to 5 bleeding events also were monitored continuously by the data safety and monitoring committee, and the difference between the treatment arms was assessed after every 100 patients were enrolled using one-sided Fisher's exact tests with a nominal type I error of 0.05. Suspension of the trial could be considered if there was a significant difference between the arms. Archival tumor tissue and blood samples were collected at baseline and after one cycle of therapy for future correlative studies.

\section{Treatment}

Patients were randomly assigned to receive either a platinum-containing chemotherapy doublet or a platinumcontaining chemotherapy doublet plus bevacizumab (BC). Each treatment cycle was 21 days. Patients could receive one of the following regimens according to investigator discretion before randomization: docetaxel $75 \mathrm{mg} / \mathrm{m}^{2}$ intravenously (IV) over 1 hour, day 1, followed by cisplatin $75 \mathrm{mg} / \mathrm{m}^{2}$ IV over 1 to 2 hours, day 1 , every 21 days; docetaxel $75 \mathrm{mg} / \mathrm{m}^{2} \mathrm{IV}$ over 1 hour, day 1 , followed by carboplatin area under the curve 6 over 30 minutes, day 1 , every 21 days; cisplatin $100 \mathrm{mg} / \mathrm{m}^{2}$ IV over 1 to 2 hours, day 1 , followed by FU $1,000 \mathrm{mg} / \mathrm{m}^{2} / \mathrm{d}$ as a continuous infusion $\times 4$ days every 21 days; or carboplatin area under the curve 6 over 30 minutes, day 1 , followed by FU $1,000 \mathrm{mg} / \mathrm{m}^{2} / \mathrm{d}$ as a continuous infusion $\times 4$ days every 21 days. Bevacizumab $15 \mathrm{mg} / \mathrm{kg}$ IV was given on day 1 of each cycle. All patients received prophylactic antibiotics with chemotherapy. Growth factor use was allowed per ASCO guidelines. Treatment continued until disease progression or intolerable toxicity in both arms. However, chemotherapy could be discontinued beyond the sixth cycle if maximum response was achieved (ie, no improvement in tumor measurements for two or more cycles). For patients who were randomly assigned to $\mathrm{BC}$, bevacizumab was continued until disease progression.

\section{Statistical Analyses}

The primary, intention-to-treat, analysis was based on all randomly assigned patients. Survival was defined as the time from randomization to death as a result of any cause, with follow-up censored at the date of last contact. Objective response was evaluated using the original RECIST. PFS was defined as the time from randomization to documented progression or death without progression. Patients without documented progression or death reported were censored at the time of the last documented disease evaluation. Kaplan-Meier estimates were used for event-time distributions. OS and PFS were compared using log-rank tests stratified by chemotherapy regimen, performance status, prior radiation to the head and neck, and weight loss in the past 6 months (as available in the randomization). Stratified Cox proportional hazards regression models were used to estimate hazard ratios (HRs). Adverse events, patient demographics, disease characteristics, and response rates were compared using Fisher's exact test. $P$ values are two-sided and Cls are at the 95\% level. Detailed adverse events were recorded with National Cancer Institute Common Terminology Criteria for Adverse Events (version 4.0).

\section{RESULTS}

\section{Patient Characteristics}

The study was activated in August 2008 and closed in February 2015, with a total accrual of 403 patients (200 in the chemotherapy arm and 203 in the BC arm). This final report is based on data available as of June 5,2018 . The median follow-up of alive patients was 40 months. Thirtytwo patients were ineligible; nine patients in the $B C$ arm never started protocol therapy. All 403 randomly assigned patients were included in the primary analysis. Among all patients, two (one in each arm) were documented as lost to follow-up. Figure 1 shows the CONSORT diagram of this study.

Patient demographics and disease characteristics of all randomly assigned patients at study entry are listed in Table 1 . The most common primary site was the oropharynx $(40.4 \%)$ followed by the larynx $(22.6 \%)$ and the oral cavity $(21.1 \%)$. Approximately $82 \%$ of patients had received prior radiation therapy to the head and neck, and $60 \%$ of patients had received prior chemotherapy. The majority of patients $(87.3 \%)$ received docetaxel (cisplatin and docetaxel or carboplatin and docetaxel) as the investigator's choice regimen in E1305. All baseline factors were well balanced between the two arms. Twenty-five

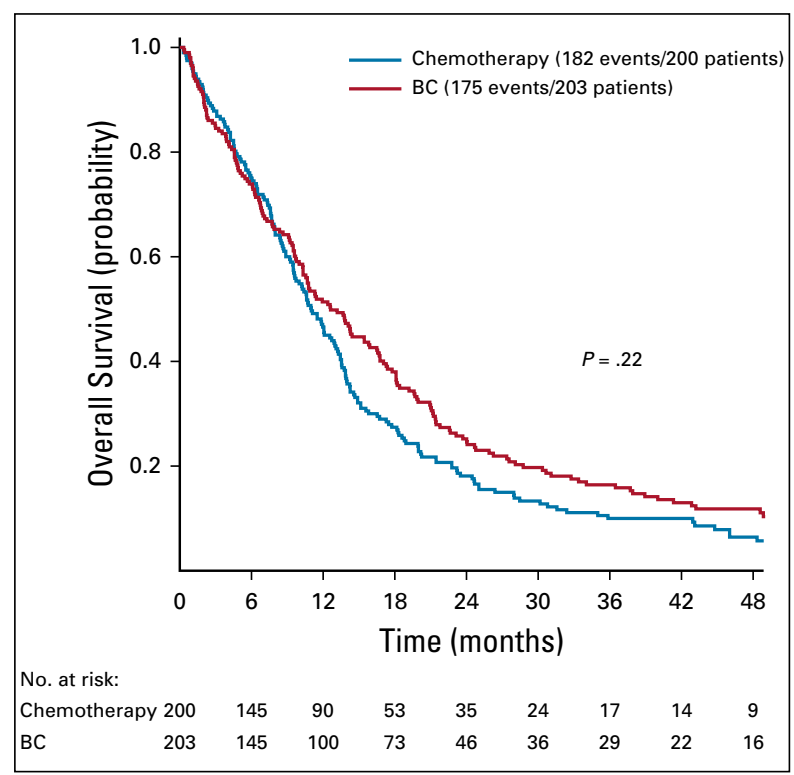

FIG 2. Kaplan-Meier estimates of overall survival by treatment arm. The median overall survival was 12.6 months with bevacizumab plus chemotherapy (BC) and 11.0 months with chemotherapy alone (hazard ratio, $0.87 ; 95 \% \mathrm{Cl}, 0.70$ to $1.09 ; P=.22$ ). 
patients (12.5\%) in the chemotherapy arm and 35 (17.2\%) in the $\mathrm{BC}$ arm had previously received cetuximab as part of multimodal treatment of locally advanced disease $(P=.21)$.

\section{Treatment Delivery}

The distribution of number of cycles reported by arm and sex is listed in Appendix Table A1 (online only). Patients who never started treatment were excluded for this analysis. The median number of treatment cycles given was four in both arms. Twenty-seven percent of patients in the BC arm received maintenance bevacizumab after six cycles, with $13 \%$ of patients receiving $>1$ year of therapy. Of the 53 patients in arm $\mathrm{BC}$ who received more than six cycles of protocol treatment, 39 received bevacizumab only, 11 received $\mathrm{BC}$, and three received chemotherapy only. Whether a patient continued protocol treatment beyond six cycles did not depend on which chemotherapy doublet they received.

\section{OS}

At the time of analysis, 357 of 403 patients in the primary analysis had died (182 in the chemotherapy arm, 175 in the $\mathrm{BC}$ arm). Figure 2 shows OS by treatment in the primary analysis. OS was not significantly different between the two arms. The median OS was 11.0 months with chemotherapy and 12.6 months with $\mathrm{BC}(P=.22)$. The estimated OS HR (BC/chemotherapy) was 0.87 (95\% Cl, 0.70 to 1.09). The 2-, 3-, and 4- OS in the $\mathrm{BC}$ versus chemotherapy arms were $25.2 \% \vee 18.1 \%, 16.4 \% \quad v$ $10.0 \%$, and $11.8 \% \vee 6.4 \%$, respectively. In an analysis that excluded patients who were either ineligible or never started protocol treatment (12 in the chemotherapy arm and 26 in the $\mathrm{BC}$ arm), the $P$ value from the log-rank test for OS was .10 with an estimated $\mathrm{HR}$ (BC/chemotherapy) of 0.82 (95\% Cl, 0.65 to 1.04$)$; the median OS in these 365 patients (188 in the chemotherapy arm and 177 in

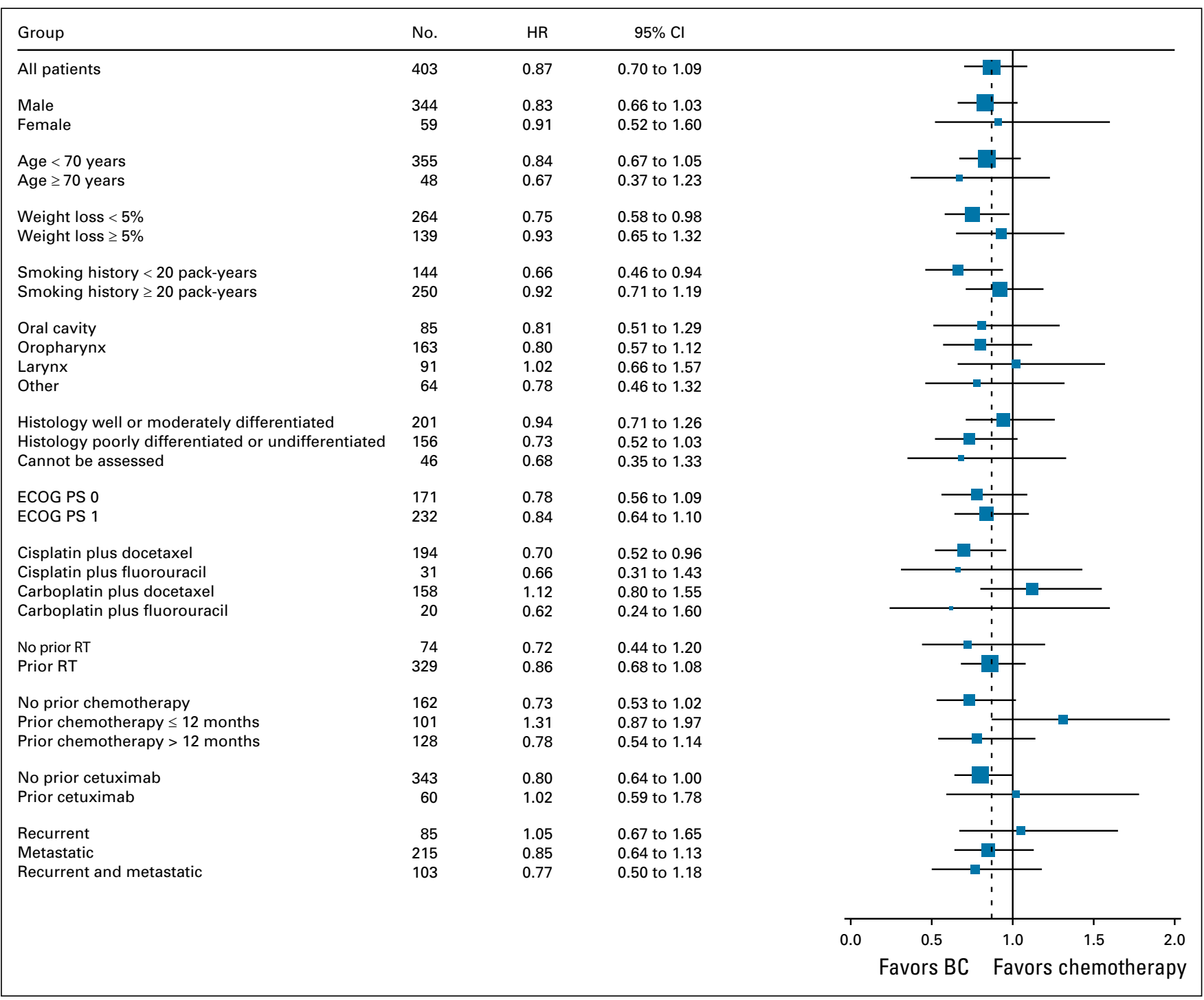

FIG 3. Forest plot of overall survival treatment hazard ratios (HRs; bevacizumab and chemotherapy [BC]/chemotherapy) in patient subgroups. ECOG PS, Eastern Cooperative Oncology Group performance status; RT, radiotherapy. 
the $\mathrm{BC}$ arm) was 11.1 months with chemotherapy and 14.2 months with BC.

Figure 3 shows a forest plot of OS for various patient subgroups. In exploratory analyses, the test for the interaction of treatment by prior chemotherapy (received $\leq 12$ months $v$ none or received $>12$ months) in a Cox proportional hazards regression model had $P=.021$, although we emphasize that this result should be interpreted with caution given the ad hoc nature of the analysis. All other interactions were not significant.

\section{PFS and Objective Response}

PFS by treatment is shown in Figure 4. The median PFS was 6.0 months with $B C$ and 4.3 months with chemotherapy $(P=.0014)$. The estimated PFS HR (BC/chemotherapy) was 0.70 (95\% Cl, 0.57 to 0.87). The 2-, 3-, and 4- PFS rates in the $\mathrm{BC}$ arm versus chemotherapy arm were $7.1 \% \vee 2.1 \%, 5.5 \% v 0.5 \%$, and $3.7 \% v 0.5 \%$, respectively. In the chemotherapy arm, only one patient (with oropharyngeal primary) achieved a 3-year PFS (censored at 48 months), whereas in the $\mathrm{BC}$ arm, 10 patients (nine with oropharyngeal primary, one with oral cavity primary) had a PFS of at least 3 years, among whom four had a PFS $>4$ years (all with oropharyngeal primary). In an analysis that excluded patients who were either ineligible or never started protocol treatment, the $P$ value from the log-rank test for PFS was $<.001$ with an estimated HR (BC/chemotherapy) of $0.68(95 \% \mathrm{Cl}, 0.53$ to 0.85$)$.

Best response by treatment is listed in Appendix Table A2 (online only). The overall response rate (complete response

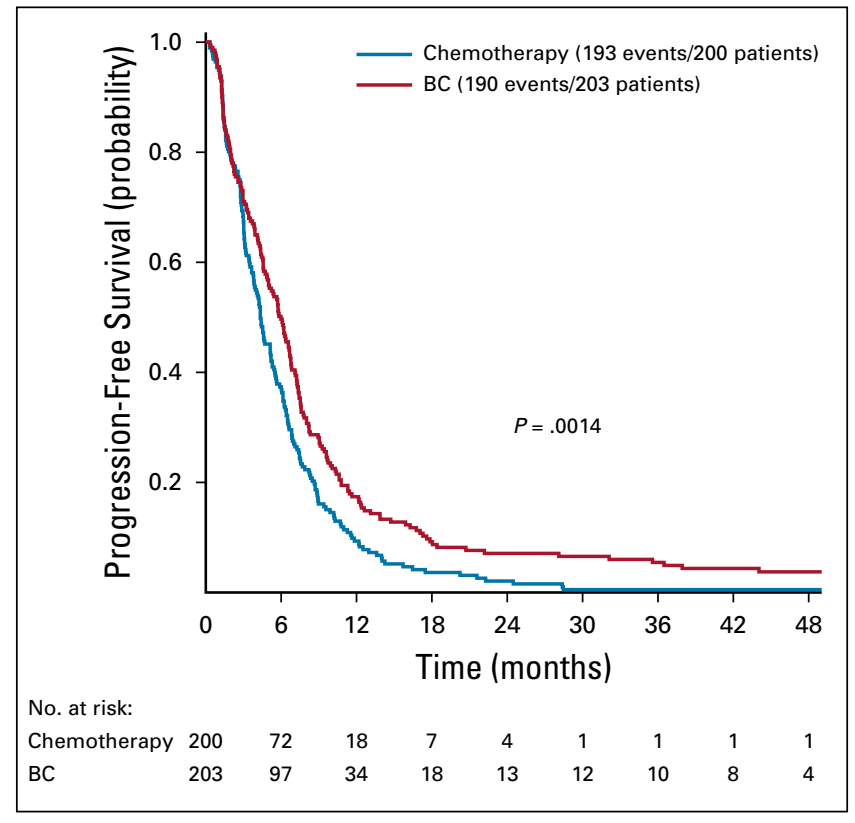

FIG 4. Kaplan-Meier estimates of progression-free survival by treatment arm. The median progression-free survival was 6.0 months with bevacizumab plus chemotherapy $(\mathrm{BC})$ and 4.3 months with chemotherapy alone (estimated hazard ratio, $0.70 ; 95 \% \mathrm{Cl}, 0.57$ to $0.87 ; P=.0014$ ). rate plus partial response rate) was higher in the $\mathrm{BC}$ arm (35.5\% with $\mathrm{BC} \vee 24.5 \%$ with chemotherapy; $P=.016)$.

\section{Toxicity}

The toxicity analysis included 392 patients (199 in the chemotherapy arm and 193 in the BC arm), excluding nine patients in the BC arm who never started treatment and two additional patients whose toxicity was not assessed (one in each arm). Treatment-related adverse events by treatment arm are listed in Table 2 (except for bleeding events, which were examined separately). Grade $\geq 3$ events were significantly higher in the $\mathrm{BC}$ arm for oral mucositis $(P<.001)$, hypertension $(P<.001)$, dehydration $(P=.001)$, thromboembolic event $(P=0.005)$, diarrhea $(P=.01)$, dysphagia $(P=.01)$, febrile neutropenia $(P=.02)$, leukopenia ( $P=.02)$, neutropenia $(P=.02)$, abdominal pain $(P=.03)$, fatigue $(P=.04)$, and worst degree of toxicity $(P<.001)$.

The incidence of grade $\geq 3$ hemorrhage and death during the study are listed in Table 3. Treatment-related grade $\geq 3$ bleeding was significantly more common with $\mathrm{BC}$ versus chemotherapy $(6.7 \% \vee 0.5 \% ; P<.001)$. There were 18 grade 5 events in the BC arm (9.3\%) and seven grade 5 events in the chemotherapy arm (3.5\%) that were classified as at least possibly a result of treatment $(P=.02)$. Of these events, grade 5 bleeding events were reported in $2.6 \%$ of patients treated with $\mathrm{BC}$ and none treated with chemotherapy. All treatment-related deaths occurred in the first 6 months.

TABLE 2. Grade 3 to 4 Toxicities (except bleeding)

\begin{tabular}{|c|c|c|c|c|}
\hline \multirow[b]{2}{*}{ Toxicity Type } & \multicolumn{2}{|c|}{$\begin{array}{l}\text { Chemotherapy } \\
(n=199), \%\end{array}$} & \multicolumn{2}{|c|}{$B C(n=193), \%$} \\
\hline & Grade 3 & Grade 4 & Grade 3 & Grade 4 \\
\hline Anemia & 8.0 & 1 & 4 & 1.0 \\
\hline Neutropenia* & 0.5 & 26 & 7 & 31.0 \\
\hline Leukopenia* & 3.0 & 14 & 5 & 22.0 \\
\hline Febrile neutropenia* & 7.0 & 3 & 11 & 7.0 \\
\hline Fatigue* & 10.0 & - & 18 & - \\
\hline Diarrhea* & 0.5 & - & 5 & - \\
\hline Dysphagia* & 0.5 & - & 5 & - \\
\hline Oral mucositis* & 4.0 & - & 19 & - \\
\hline Nausea & 8.0 & - & 11 & - \\
\hline Vomiting & 5.0 & - & 7 & 0.5 \\
\hline Anorexia & 7.0 & - & 6 & - \\
\hline Abdominal pain* & 0.5 & & 4 & \\
\hline Dehydration* & 6.0 & - & 15 & 0.5 \\
\hline Hypertension* & - & - & 6 & - \\
\hline Thromboembolic event* & 0.5 & - & 5 & 0.5 \\
\hline Worst degree* $\dagger$ & 31.0 & 34 & 34 & 41.0 \\
\hline
\end{tabular}

Abbreviation: BC, bevacizumab plus chemotherapy. ${ }^{*} P<.05$ for the comparison between the two arms. †Worst degree toxicity includes bleeding. 
TABLE 3. Bleeding and Grade 3 to 5 Toxicity Per Arm

\begin{tabular}{|c|c|c|c|c|c|c|}
\hline \multirow[b]{2}{*}{ Grade } & \multicolumn{3}{|c|}{ Treatment-Related Adverse Events, No. (\%) } & \multicolumn{3}{|c|}{ All Reported Adverse Events, No. (\%) } \\
\hline & Chemotherapy & BC & $P$ & Chemotherapy & BC & $P$ \\
\hline All grade 5 & $7(3.5)$ & $18(9.3)$ & .022 & $41(20)$ & $46(22.7)$ & .54 \\
\hline All grade $3-5$ & $135(67)$ & $162(82)$ & $<.001$ & $161(79)$ & $167(85.1)$ & .12 \\
\hline Grade 5 bleeding & $0(0)$ & $5(2.6)$ & .028 & $2(1.0)$ & $7(3.6)$ & .10 \\
\hline Grade $3-5$ bleeding & $1(0.5)$ & $13(6.7)$ & $<.001$ & $7(3.5)$ & $15(7.7)$ & .08 \\
\hline
\end{tabular}

Abbreviation: BC, bevacizumab plus chemotherapy.

\section{DISCUSSION}

This was a large, phase III, multicenter trial in the first-line treatment setting of patients with recurrent or metastatic SCCHN. The addition of bevacizumab to a platinum-based doublet did not improve OS but did improve PFS and response rate. The median survival was 12.6 months in the $\mathrm{BC}$ arm and 11.0 months in the chemotherapy arm (HR, $0.87 ; 95 \% \mathrm{Cl}, 0.70$ to $1.09 ; P=.22$ ). Although a statistically significant improvement in OS was not reached, there was noted numerical survival advantage at 2, 3, 4, and 5 years in the BC arm (25.2\% v 18.1\%, 16.4\% v 10.0\%, 11.8\% v $6.4 \%$, and $7.2 \% \vee 4.0 \%$, respectively). Moreover, median PFS improved from 4.3 to 6.0 months (HR, 0.70; $P=$ $.0014)$, and the response rate increased from $24.5 \%$ to $35.5 \%(P=.016)$ with BC. Survival in the chemotherapy arm was markedly better than expected, which could be explained by various factors, including the use of docetaxel in the regimen, increased incidence of human papillomavirus-associated oropharyngeal tumors, improvements in supportive care, and the potential impact of subsequent lines of therapy. Cetuximab became US Food and Drug Administration approved for recurrent or metastatic SCCHN during this study. We did not collect subsequent treatment information, and whether second-line cetuximab played a role in the OS results reported cannot be assessed. Moreover, we note that as in most other bevacizumab randomized trials, this was not a placebocontrolled trial. Whether the lack of placebo affected any of the efficacy parameters examined or the toxicity reporting is not possible to ascertain.

Despite our careful exclusion criteria aimed at reducing the chances of treatment-related bleeding and the use of antibiotic prophylaxis, we observed a significant increase in treatment-related toxicities with bevacizumab, including grade 3 to 5 bleeding, oral mucositis, dysphagia, diarrhea, fatigue, febrile neutropenia, and thromboembolic events. It is possible that the increased number of treatment-related deaths in the BC arm that occurred in the first 6 months affected our ability to demonstrate a survival difference between the two arms. After the first few months, the OS curves separated in favor of the BC arm, and at 3 and 4 years, there were approximately twice as many long-term survivors with $\mathrm{BC}$ versus chemotherapy alone. Given this encouraging finding as well as the better PFS and response rates with the addition of bevacizumab to standard chemotherapy doublets, the VEGF pathway appears to be a relevant therapeutic target in SCCHN. Better tolerated anti-VEGF pathway agents that are not associated with high rates of bleeding may be considered in future clinical trials in SCCHN. Individualized anti-angiogenic treatment on the basis of molecular tumor characterization is desirable and may lead to improved outcomes. Because tissue and blood samples have been collected in the context of E1305, this will be the subject of future analysis from our group.

In conclusion, this study indicates that the addition of bevacizumab to chemotherapy improves PFS and response rates but increases toxicities in the first-line treatment of patients with recurrent or metastatic SCCHN. We observed a small, but not statistically significant, improvement in OS. Although the study may have been underpowered because of the longer survival in the chemotherapy arm, the identification of patient characteristics and biomarkers for survival benefit will be critical for further development of this strategy in SCCHN. On the basis of the results of E1305, we believe that the targeting of angiogenesis remains an attractive strategy, and further investigation of agents that target the VEGF pathway in SCCHN is justifiable.

\section{AFFILIATIONS}

${ }^{1}$ Thomas Jefferson University, Philadelphia, PA

${ }^{2}$ Dana Farber Cancer Institute, Boston, MA

${ }^{3}$ University of Arizona Cancer Center, Phoenix, AZ

${ }^{4}$ University of Pittsburgh, Pittsburgh, PA

${ }^{5}$ Vanderbilt University, Nashville, TN

${ }^{6}$ Greater Baltimore Medical Center, Baltimore, MD

${ }^{7}$ The Ohio State University Medical School, Columbus, $\mathrm{OH}$

${ }^{8}$ Montefiore Medical Center, Bronx, NY

\footnotetext{
${ }^{9}$ Emory University, Atlanta, GA

${ }^{10}$ University of Miami, Miami, FL

${ }^{11}$ University of Pennsylvania, Philadelphia, PA

${ }^{12}$ Stanford University Medical Center, Stanford, CA

${ }^{13}$ Johns Hopkins University, Baltimore, MD

${ }^{14}$ Yale University School of Medicine, New Haven, CT

${ }^{15}$ Yale Cancer Center, New Haven, CT
} 


\section{CORRESPONDING AUTHOR}

Athanassios Argiris, MD, Thomas Jefferson University, 1025 Walnut St, Suite 700, Philadelphia, PA 19107; e-mail: athanassios.argiris@ gmail.com.

\section{PRIOR PRESENTATION}

Presented at the American Society of Clinical Oncology 2015 Annual Meeting, Chicago, IL, May 29-June 2, 2015, and the American Society of Clinical Oncology 2017 Annual Meeting, Chicago, IL, June 2-7, 2017.

\section{SUPPORT}

Supported by the National Cancer Institute under the following award numbers: CA180820, CA180794, CA180802, CA180816, CA180826, CA180847, CA180853, CA180864, CA180868, CA189859, CA180844, and CA189819.

This study was coordinated by the ECOG-ACRIN Cancer Research Group (Peter J. O'Dwyer, MD, and Mitchell D. Schnall, MD, PhD, group cochairs).

The content is solely the responsibility of the authors and does not necessarily represent the official views of the National Institutes of Health, nor does mention of trade names, commercial products, or organizations imply endorsement by the US government.
AUTHORS' DISCLOSURES OF POTENTIAL CONFLICTS OF INTEREST AND DATA AVAILABILITY STATEMENT

Disclosures provided by the authors and data availability statement (if applicable) are available with this article at DOI https://doi.org/10.1200/ JC0.19.00555

\section{AUTHOR CONTRIBUTIONS}

Conception and design: Athanassios Argiris, Shuli Li, Arlene A. Forastiere, Barbara Burtness

Administrative support: Athanassios Argiris, Panayiotis Savvides, Barbara Burtness

Provision of study material or patients: Athanassios Argiris, Panayiotis

Savvides, Jill Gilbert, Marshall A. Levine, Missak Haigentz Jr, Charles J. Schneider, Harlan A. Pinto, Arlene A. Forastiere, Barbara Burtness

Collection and assembly of data: Athanassios Argiris, Shuli Li, Panayiotis Savvides, James P. Ohr, Marshall A. Levine, Arnab Chakravarti, Nabil F. Saba, Chukwuemeka V. Ikpeazu, Charles J. Schneider, Harlan A. Pinto, Barbara Burtness

Data analysis and interpretation: Athanassios Argiris, Shuli Li, Jill Gilbert, Marshall A. Levine, Arnab Chakravarti, Missak Haigentz Jr, Nabil F. Saba, Barbara Burtness

Manuscript writing: All authors

Final approval of manuscript: All authors

Accountable for all aspects of the work: All authors

\section{CLINICAL TRIAL INFORMATION}

Clinical trial information: NCT00588770.

\section{REFERENCES}

1. Argiris A, Karamouzis MV, Raben D, et al: Head and neck cancer. Lancet 371:1695-1709, 2008

2. Gibson MK, Li Y, Murphy B, et al: Randomized phase III evaluation of cisplatin plus fluorouracil versus cisplatin plus paclitaxel in advanced head and neck cancer (E1395): An intergroup trial of the Eastern Cooperative Oncology Group. J Clin Oncol 23:3562-3567, 2005

3. Vermorken JB, Mesia R, Rivera F, et al: Platinum-based chemotherapy plus cetuximab in head and neck cancer. N Engl J Med 359:1116-1127, 2008

4. Vassilakopoulou M, Psyrri A, Argiris A: Targeting angiogenesis in head and neck cancer. Oral Oncol 51:409-415, 2015

5. Mineta H, Miura K, Ogino T, et al: Prognostic value of vascular endothelial growth factor (VEGF) in head and neck squamous cell carcinomas. Br J Cancer 83 : 775-781, 2000

6. Kyzas PA, Cunha IW, loannidis JP: Prognostic significance of vascular endothelial growth factor immunohistochemical expression in head and neck squamous cell carcinoma: A meta-analysis. Clin Cancer Res 11:1434-1440, 2005

7. Fujita K, Sano D, Kimura M, et al: Anti-tumor effects of bevacizumab in combination with paclitaxel on head and neck squamous cell carcinoma. Oncol Rep 18: 47-51, 2007

8. Jain RK: Normalizing tumor microenvironment to treat cancer: Bench to bedside to biomarkers. J Clin Oncol 31:2205-2218, 2013

9. Argiris A, Karamouzis MV, Gooding WE, et al: Phase II trial of pemetrexed and bevacizumab in patients with recurrent or metastatic head and neck cancer. J Clin Oncol 29:1140-1145, 2011

10. Argiris A, Kotsakis AP, Hoang T, et al: Cetuximab and bevacizumab: Preclinical data and phase II trial in recurrent or metastatic squamous cell carcinoma of the head and neck. Ann Oncol 24:220-225, 2013

11. Cohen EE, Davis DW, Karrison TG, et al: Erlotinib and bevacizumab in patients with recurrent or metastatic squamous-cell carcinoma of the head and neck: A phase I/II study. Lancet Oncol 10:247-257, 2009

12. Argiris A, Li Y, Forastiere A: Prognostic factors and long-term survivorship in patients with recurrent or metastatic carcinoma of the head and neck. Cancer 101 : 2222-2229, 2004 
Phase III Randomized Trial of Chemotherapy With or Without Bevacizumab in Patients With Recurrent or Metastatic Head and Neck Cancer

The following represents disclosure information provided by authors of this manuscript. All relationships are considered compensated unless otherwise noted. Relationships are self-held unless noted. I = Immediate Family Member, Inst = My Institution. Relationships may not relate to the subject matter of this manuscript. For more information about ASCO's conflict of interest policy, please refer to www.asco.org/rwc or ascopubs.org/jco/site/ifc.

Open Payments is a public database containing information reported by companies about payments made to US-licensed physicians (Open Payments).

\section{Athanassios Argiris}

Consulting or Advisory Role: Merck Serono, Bristol-Myers Squibb, Roche, Debiopharm Group, Aspyrian Therapeutics

Speakers' Bureau: Merck Serono, Bristol-Myers Squibb

Research Funding: Genentech, Roche, Bristol-Myers Squibb

Travel, Accommodations, Expenses: Bristol-Myers Squibb, Merck Serono

Panayiotis Savvides

Consulting or Advisory Role: Heron, Bayer AG, Regeneron Pharmaceuticals, Bristol-Myers Squibb, Genzyme

Speakers' Bureau: Regeneron Pharmaceuticals, Bristol-Myers Squibb, Genzyme

Research Funding: Regeneron Pharmaceuticals (Inst), Novartis (Inst)

Jill Gilbert

Leadership: American Board of Internal Medicine, Tennessee Oncology Practice Society

Honoraria: Sanofi, UpToDate, TRM Oncology, Bayer AG

Consulting or Advisory Role: EPIC, IRX Therapeutics, Sanofi, ASCO University, Bayer AG, CUE Biopharma

Research Funding: AstraZeneca (Inst), Bristol-Myers Squibb, Threshold Pharmaceuticals, Pfizer, Merck, Amgen (Inst)

Marshall A. Levine

Stock and Other Ownership Interests: Gilead, Illumina, Pfizer, Amgen

\section{Missak Haigentz Jr}

Consulting or Advisory Role: AstraZeneca, Takeda Pharmaceuticals, Genentech

\section{Nabil F. Saba}

Honoraria: Merck, Eli Lilly, Pfizer, Bristol-Myers Squibb, CUE Biopharma, GlaxoSmithKline, Aduro Biotech, Kura Oncology, Genentech, Roche, Pfizer, Bristol-Myers Squibb, Merck

Research Funding: Bristol-Myers Squibb, Exelixis

Travel, Accommodations, Expenses: Bristol-Myers Squibb, Merck, Pfizer, Eli Lilly, GlaxoSmithKline, Genentech, Roche

Chukwuemeka V. Ikpeazu

Consulting or Advisory Role: AstraZeneca, Bayer AG

Research Funding: AstraZeneca, Genentech, Roche, Merck, Eisai

\section{Barbara Burtness}

Consulting or Advisory Role: Merck, Medlmmune, Debiopharm Group, VentiRx, AstraZeneca, Bristol-Myers Squibb, Alligator Biosciences, Genentech, Roche, Aduro Biotech, GlaxoSmithKline, Celgene, CUE Biopharma, Maverick Therapeutics, Rakuten, Nanobiotix, Bayer AG

Travel, Accommodations, Expenses: Merck, Debiopharm Group, Boehringer Ingelheim

No other potential conflicts of interest were reported. 


\section{APPENDIX}

TABLE A1. Number of Cycles Delivered in Each Arm

\begin{tabular}{lcc} 
Cycles, No. & Chemotherapy $(\mathbf{n}=\mathbf{2 0 0})$, No. $(\%)$ & BC $(\mathbf{n}=\mathbf{2 0 3})$, No. (\%) \\
\hline 1 & $25(12.5)$ & $27(13.9)$ \\
\hline 2 & $32(16)$ & $32(16.5)$ \\
\hline 3 & $8(4)$ & $19(9.8)$ \\
\hline 4 & $36(18)$ & $24(12.4)$ \\
\hline 5 & $13(6.5)$ & $13(6.7)$ \\
\hline 6 & $65(32.5)$ & $26(13.4)$ \\
\hline $7-10$ & $21(10.5)$ & $28(14.4)$ \\
\hline $11-15$ & $0(0)$ & $11(5.6)$ \\
\hline $17-20$ & $0(0)$ & $9(4.5)$ \\
\hline $23-54$ & $0(0)$ & $5(2.5)$
\end{tabular}

Abbreviation: $\mathrm{BC}$, bevacizumab plus chemotherapy.

TABLE A2. Best Objective Response by Treatment Arm

Chemotherapy $(n=200)$,

No. (\%)

BC $(n=203)$, No. $(\%)$

\begin{tabular}{lll} 
Response & \multicolumn{1}{c}{ No. (\%) } & BC (n= 203), No. (\%) \\
\hline $\begin{array}{l}\text { Overall response rate (complete response plus } \\
\text { partial response), \% }(95 \% ~ C l)^{*}\end{array}$ & $24.5(18.7$ to 31.0) & $35.5(28.9$ to 42.5$)$ \\
\hline Complete response & $2(1.0)$ & $11(5.4)$ \\
\hline Partial response & $47(23.5)$ & $61(30.1)$ \\
\hline Stable disease & $75(37.5)$ & $58(28.6)$ \\
\hline Progression & $39(19.5)$ & $27(13.3)$ \\
\hline Unevaluable & $37(18.5)$ & $46(22.7)$
\end{tabular}

Abbreviation: $\mathrm{BC}$, bevacizumab plus chemotherapy.

${ }^{*} P=.016$ for the comparison between the two arms. 